\title{
BP-SY-3-4
}

\section{Application of artificial intelligence in surgery}

\author{
In Woong HAN*
}

Department of Surgery, Samsung Medical Center, Sungkyunkwan University School of Medicine, Seoul, Korea

Lecture: Machine-learning (ML) is an artificial intelligence (AI) technology that has been adopted in many areas of modern society, including medical science. In ML, computational models composed of multiple processing layers learn various data representations with multiple levels of abstraction. ML is currently being used in not only surgery but also other areas, such as, pharmacogenomics, image classification, and medical decision support systems. Therefore, for this lecture, I aim to summarize various new risk prediction platforms after surgery using ML algorithms. If so, we would expect that a patient's predicted various risk after surgery could direct their clinical management and prevent or mitigate untoward outcomes. 